\title{
Improving Innovation in Nano-Healthcare Funding
}

\author{
Mike Eaton* \\ ENATRANS, c/o TecMinho, Campus de Azurém da Universidade do Minho, 4800-058, Guimarães, \\ Portugal
}

Submitted: January 11, 2018

Accepted: February 18, 2018

Published April 17, 2018

\section{Introduction and Discussion}

European countries have invested heavily in Nanomedicine over the last decade, however the output has been much reduced by a lack of knowledge of how to innovate in a heavily regulated setting $[1,2]$. This development failing is not unique to nanomedicine but is there to differing extents across most open innovation healthcare projects.

The healthcare value of this public funding has been shrunk by the beneficiaries' lack of understanding of development - not knowledge of research $[3,4]$. Public funding systems assume this development knowledge is understood by both scientists and peer review funders neither is true, which reduces funding to an expensive lottery (Figure 1). Successful translation requires a detailed understanding of the path through and beyond the clinic to the market, with a quantifiable value to patients, companies, innovators, tax payers and funders. Healthcare is rightly a highly-regulated sector with a slow and extremely expensive development process; it is not an area to enter lightly unless you have development knowledge and a strong Unique Selling Proposition (USP). In any specific therapeutic area - unlike most business areas - there is only a market for a few products, due to the development costs. Development processes and their milestones are not obscure, but it does require effort to learn and practice them, and these essential skills are not taught at present. There are early plans to teach healthcare product development in some European countries, but these are at a pilot or low level at present.

\footnotetext{
*Email: nanomedicine@btinternet.com
}

Universities' philosophy is to create globally competitive research centres; however, they very rarely train students or recruit lecturers with a background in development science or thinking. Their staff, in due course, inevitably view healthcare from their narrow research perspective when invited to peer review (de facto "applied") projects. There is a big opportunity here for European Science to encompass development science(s) but this change from the status quo lacks motivation? Without high level political encouragement, the situation will continue to help a minority, and not the stakeholders that matter.

The consequences of this research-focused culture are that many applied projects at inception start with a very low chance of success, from a development and patient perspective; again, not surprisingly, many such projects are publicly funded at a serious level by "expert" academic pee reviewers. If you want to build a house do you employ an experienced builder or an expert in cement technology?

The ground rules for development have been published and are freely available [3, 4]. The industry development milestones and relevant gatekeeping questions are openly used by ENATRANS5, an EC funded translation advisory service, as it evaluates and supports innovation in academia and industry.

Some of the problems seen by ENATRANS can be solved by training on how to showcase projects to an industrial audience. 


\section{Research Knowledge is not Development Knowledge}

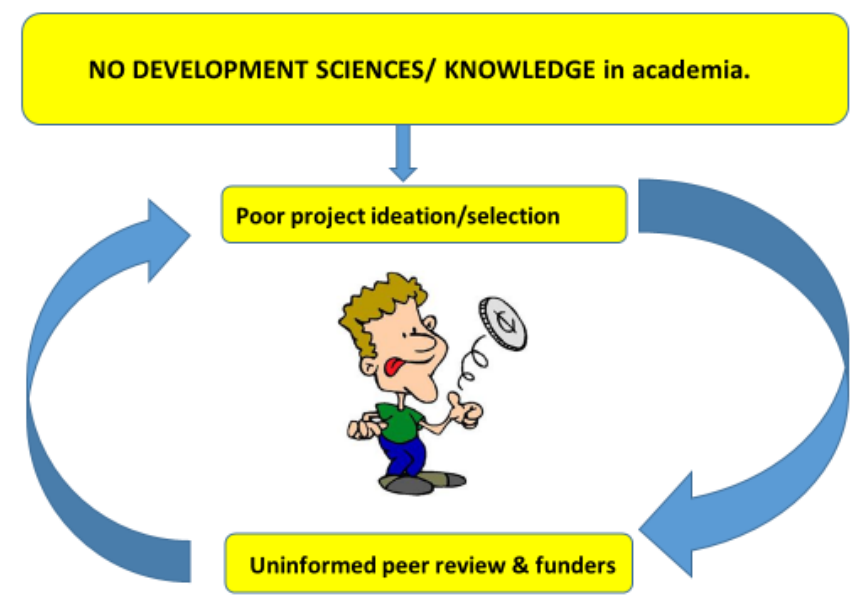

Figure 1 The lack of development know-how leads to a lottery in healthcare funding

This requires joined up thinking, and a clear presentation, demonstrating the advantages of their chosen approach compared with a competitor. There are many opportunities for a strong technology transfer office to advise on translation, but this rarely happens.

ENATRANS has seen exceptional developable projects, but the majority are industry-benchmarked as very early research, and often lack the industrial metrics for acceptance into development. Whilst ENATRANS's main role was envisaged as assisting in the preclinical stage, it has de facto assumed an educational role, as well in helping at ideation, clearly signaling an unmet need. Some projects reach ENATRANS without funding and others have been funded substantially, without addressing development metrics such as "freedom to operate". It has been said that researchers in nanomedicine are unable to address industrial norms in the $\mathrm{R}$ to $\mathrm{D}$ transition - however in the author's experience researchers CAN acquire these skills even to a high level quite quickly if it makes a funding difference.

The current culture helps academics, but does not make Europe more competitive, nor does it help taxpayers or patients. Cultural change is difficult, but this is truly a win-win-win opportunity for researchers as their eyes and careers can be opened to the real world and the difficult challenges in healthcare, including the "blue sky" ones. Incremental or derivative research is the fallback of the research community, but in comparison to radical innovation, it leads to a product concept with a lower USP. A step change in creativity is required for radical innovation, but if successful will have a stronger USP. Such a step change led to the birth of Biologicals - such innovation is rare but repeatable.

The lever for change lies crucially in the hands of funders, as they fund and direct scientists either to healthcare research or development. Funding should not be automatic through $\mathrm{R}$ to $\mathrm{D}$, because the transition from research to development requires informed debate and high-quality data and is a very challenging milestone. Researchers often say they are developing a new drug, when they are in fact doing research - funders also use the terms ( $\mathrm{R}$ or $\mathrm{D})$ interchangeably - an unfortunate consequence of their academic training. A simple test is if you don't know actually what you are developing - you are in research. 
Both terms should be used appropriately and remember, development is not reversible due to its expensive resource requirements. Development costs are so high and timeconsuming, that it permits only a handful of drugs, to be marketed for any indication. Development also necessitates a knowledge not only of current competitors, but of future markets - a good USP is everything. Whilst a researchbased model works well for research, it needs to be expanded to make it more appropriate for healthcare development. It is a serious flaw to start a research project with no knowledge of the ultimate development requirements.

\section{Conclusion}

In conclusion, the areas for improvement are:

- Knowledgeable peer review to select the most promising research and development healthcare topics for funding.

- A process for development scientists / entrepreneurs to train researchers/students.

- A more pro-active, informed role for "Technology" Transfer Officers/Business Development officers.

- Learning Communication skills for entry into development. A shift from technologies to products with commercial or societal value.

\section{Conflict of interest}

The author declares no relevant interest to disclose. For a signed statement contact the journal office editor@precisionnanomedicine.com.

\section{References}

[1] M. Eaton, "Nanomedicine: Industry-wise research," Nature Materials, vol. 6, no. 4, pp. 251-253, 1 April 2007.

[2] M. A. Eaton, "Improving the translation in Europe of nanomedicines (a.k.a. drug delivery) from academia to industry," Journal of Controlled Release, vol. 164, no. 3, pp. 370-337, 28 December 2012.

[3] E. H. Chang, J. B. Harford, M. A. Eaton, P. M. Boisseau, A. Dube, R. S. H. Hayeshi and D. S. Lee, "Nanomedicine: Past, present and future - A global perspective," Biochemical and Biophysical Research Communications, vol. 468, no. 3, pp. 511-517, 18 December 2015.

[4] M. A. Eaton, L. Levy and O. M. Fontaine, "Delivering Nanomedicines to Patients: A Practical Guide," Nanomedicine: Nanotechnology, Biology and Medicine, vol. 11, no. 4, pp. 983-992, May 2015.

[5] "ENATRANS - Enabling Nanomedicine Translation," [Online]. Available: http://www.enatrans.eu/public. [Accessed 1 February 2018].

Quote this article as Eaton, M., Improving Innovation in Nano-Healthcare Funding, Precis. Nanomed. 2018;1(1):15-17, https://doi.org/10.29016/180108.1 